\title{
Dati DOPPS e dose dialitica: considerazioni assistenziali
}

\author{
M. Pegoraro
}

EDTNA/ERCA Filiale Italiana, Milano

\section{Introduzione}

Il concetto di corretta dose dialitica si è strutturato ed empiricamente formulato nel tempo a partire dalla metà degli anni Ottanta (1). Il KT/V è un dato trovato dai professionisti per indirizzare la prescrizione secondo dati oggettivi e su questi confrontare gli esiti. L'uso dello strumento KT/V è diventato nel tempo sempre più comune e ha potuto essere usato per confrontare studi (2-4) e monitorare i cambiamenti nelle pratiche cliniche a livello internazionale, rispetto alle aderenze alle linee guida (LG), e conseguentemente poter correlare il suo andamento agli esiti sui pazienti (Fig. 1).

In questo articolo vedremo come il KT/V è il condensato matematico di molte variabili, che associate a sesso, età, e abitudini di vita dei pazienti, entrano nella valutazione dell'adeguatezza dialitica e possono incidere nella definizione di dose ottimale per ogni soggetto.

Ricordiamo che il KT/V adeguato è definito come un valore superiore a 1,2 se in comparto unico (single/ pool) $0>1,05$, se inteso come valore matematicamente equilibrato $(\mathrm{eKT} / \mathrm{V})$, che tiene conto della disposizione pluricompartimentale dei liquidi corporei.

\section{Cosa include il KT/V}

K: clearance, depurazione. Solitamente si utilizza il valore dell'urea ematica come indicatore, ma la formula può essere utilizzata anche per altri soluti. Il valore di urea pre-dialitica deve essere ovviamente maggiore del valore post-dialitico. L'ampiezza della differenza tra i valori di inizio e fine dialisi è correlata alle variabili di dimensione del filtro utilizzato e alla quantità di litri di sangue dializzato. La clearance dell'urea è maggiore nelle prime due ore e diminuisce di efficacia con l'avanzare della dialisi. Questa perdita di efficienza è imputabile alla via via ridotta disponibilità ematica o tissutale dell'urea all'avvicinarsi dei tassi di concentrazione fisiologici della stessa o quando i meccanismi di richiamo cellulare sono rallentati, o anche e, per minima parte, dall'esaurimento della membrana, se l'anticoagulazione è ottimale. $\mathrm{Ci}$ sono sostanze viceversa la cui clearance aumenta con la durata della dialisi, perché queste devono liberarsi dai legami proteici o enzimatici, come nel caso del fosforo. La clearance di altre sostanze sarà viceversa proporzionale alla permeabilità della membrana, come per esempio per la Beta2 globulina. Alcuni elettroliti come il calcio (Ca) e i bicarbonati $\left(\mathrm{HCO}_{3}\right)$ hanno un andamento opposto, per effetto della correzione verso il dato fisiologico. Il valore dell'urea nel sangue ha origine per 1' $80 \%$ da introiti azotati (proteine alimentari) e per il 20\% da metabolismo endogeno. Una bassa urea pre-dialisi può essere indicatore di scarsa nutrizione, a meno che il paziente non abbia ancora una buona diuresi residua (5). Nell'ambito della formula, $\mathbf{K}$ può essere modificato dalla scelta del filtro utilizzato e dalle caratteristiche che le diverse membrane esprimono in termini di coefficiente di depurazione $(\mathrm{KoA})$.

T: tempo. Espresso negli studi DOPPS in minuti, definisce la durata della dialisi: cioè il tempo necessario per permettere il passaggio dal sangue al dialisato, della quantità di metaboliti che devono essere rimossi/riequilibrati per mantenere una corretta omeostasi. L'elemento tempo cela il concetto di efficacia. A parità di filtro, un'ora di trattamento con un flusso sangue $(\mathrm{Qb})$ di 350 $\mathrm{ml} / \mathrm{min}$, depura più sangue di un'ora con Qb di $250 \mathrm{ml} /$ min. Per questa ragione in alcuni Centri, ed ora in alcune metodiche di trattamento, l'elemento "durata della dialisi” è subordinato alla quantità di litri depurati (6). Il raggiungimento di un certo quantitativo di litri depurati (sia facendo riferimento al sangue, oppure al dialisato), rappresentano la dose di dialisi. Il $\mathrm{Qb}$ è primariamente connesso con il tipo e portata dell'accesso vascolare, nonché alle condizioni generali del paziente. Per un pa- 


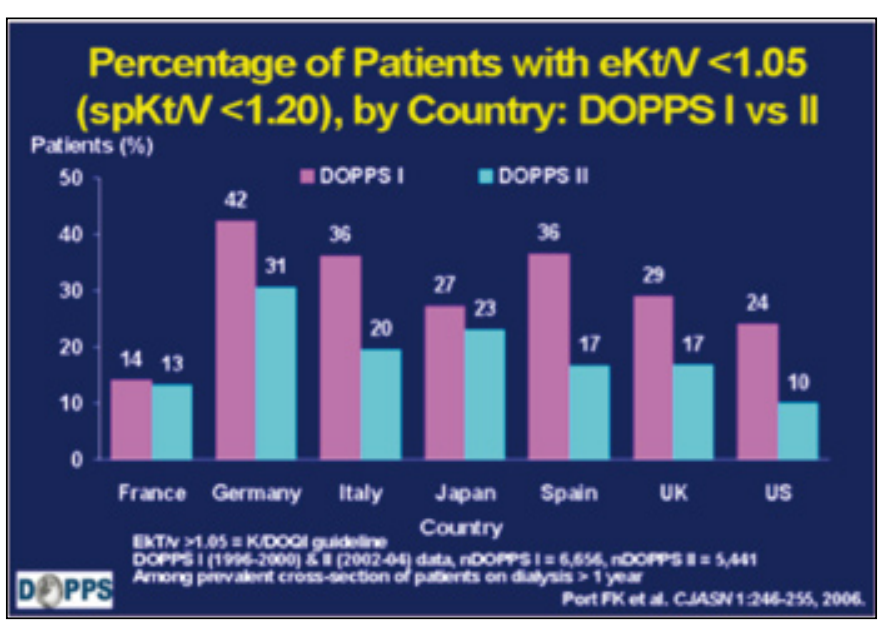

Fig. 1 - Percentuale di pazienti che nei periodo di Dopps 1 e Dopps 2 NON erano complianti con le indicazioni del KT/date dei KDOQI.

ziente agli inizi del suo trattamento dialitico in genere si utilizza un $\mathrm{Qb}$ minore, sia per la frequente scarsa maturazione della FAV o per la sua frequente maggiore fragilità, sia per evitare le sindromi da squilibrio elettrolitico, di possibile più frequente riscontro nelle prime dialisi specie in pazienti late-referral. Per questo motivo alcuni dei dati DOPPS hanno valori calcolati in base alla permanenza in dialisi dei soggetti studiati ed alcuni studi DOPPS hanno mostrato un progressivo relativo aumento della durata della seduta emodialitica, soprattutto in alcune aree geografiche. Ovviamente quando i valori ematici dei metaboliti tossici accumulati sono elevati ovvero se l'aumento interdialitico è eccessivo, se il paziente ha una ampia taglia (espressa come indice di massa corporea $\mathrm{o} \mathrm{BMI})$, la durata della dialisi dovrà essere teoricamente maggiore che in un paziente di bassa taglia e/o il $\mathrm{Qb}$ adoperato nello spazio temporale dovrà essere più elevato. La variabile "tempo" è la variabile più subdola, e la sua alterazione è cosa facile. A volte lo richiede il paziente, altre volte le esigenze tempistiche del reparto, a volte sorgono dei problemi di accesso vascolare ecc. $\mathrm{E}$ fondamentale avere coscienza (e instillarla nei pazienti) che il "tempo" è un elemento che ha stretta correlazione con la dose dialitica. Ciò appare ovvio per gli operatori di dialisi, ma non lo è spesso per i pazienti. Il tempo non è un valore assoluto - come lo sono invece il volume di distribuzione, o il filtro in uso - bensì il tempo è un valore relativo da porre in relazione $\mathrm{al} \mathrm{Qb}$ in uso e quindi se, $\mathrm{ad}$ esempio, per più dialisi dovesse persistere un basso $\mathrm{Qb}$, si può rendere necessario l'incremento della variabile $\mathrm{T}$ sino alla programmazione di dialisi supplementari.

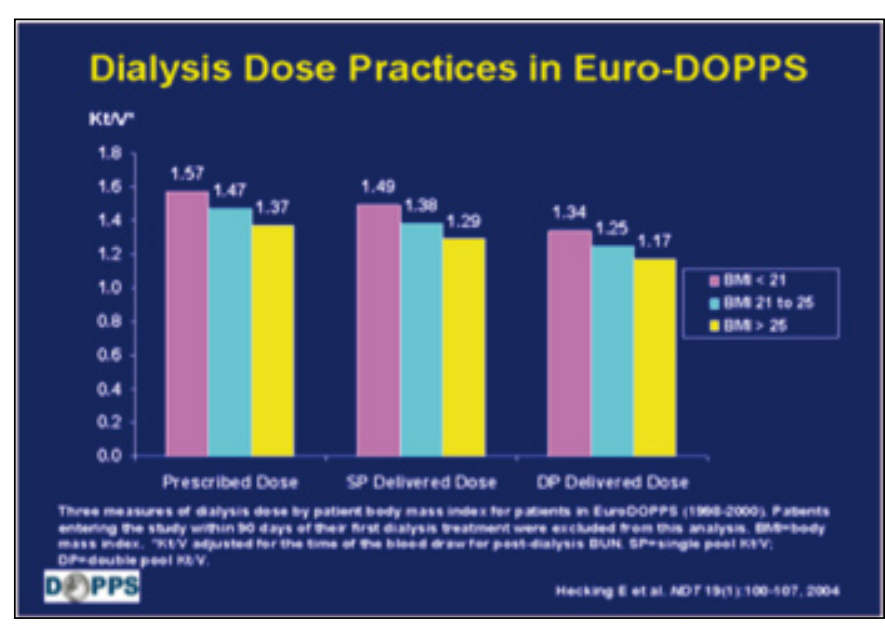

Fig. 2 - Caratteristiche della dose dialitica nei Paesi europei in relazione all'indice di massa corporea (BMI).

V: volume da depurare. Esprime il volume corporeo in cui sono distribuiti i metaboliti. Più è grande il soggetto da depurare, maggiore sarà la quantità di metaboliti da depurare. Solitamente V si esprime con il Volume di Distribuzione (VD), espresso in un numero ricavato utilizzando la formula di Watson* (1) che tiene conto del peso, dell'altezza e nel maschio anche dell'età. Il tutto moltiplicato per coefficienti stabiliti. Approssimativamente potremmo dire che in un paziente maschio adulto, al crescere del BMI e al diminuire dell'età, maggiore sarà la necessità di depurazione rispetto a un paziente di sesso femminile e di piccola taglia. Ovviamente ciò non riveste una regola matematica bensi proporzionale in senso lato: ad esempio a un paziente che pesa $90 \mathrm{~kg}$ non faremo una dialisi di doppia durata rispetto a quella dovuta a una paziente che pesa solo $45 \mathrm{~kg}$. Per ovviare a tali differenze si usa modificare le altre variabili come l'aumento della superficie del filtro o della permeabilità dello stesso, l'applicazione di metodiche non solo diffusive ma anche convettive, la variazione all'aumento dei flussi (sia del sangue o $\mathrm{Qb}$ sia dei fluidi di dialisi), sino ovviamente a modificare anche la variabile $\mathrm{T}$, ovvero aumentando la durata della singola seduta o il numero delle sedute settimanali.

Tutto ciò spiega perché nei dati DOPPS sono emersi diversi valori tra i vari Paesi partecipanti. Per esempio, i

\footnotetext{
*Formula di Watson: uomini: $2,447-\left(0,095^{\star}\right.$ età $)+\left(0,107^{\star}\right.$ altezza $)+\left(0,336^{*}\right.$ peso $)$ donne: $2,097+\left(0.107^{\star}\right.$ altezza $)+\left(0,247^{\star}\right.$ peso $)$
} 


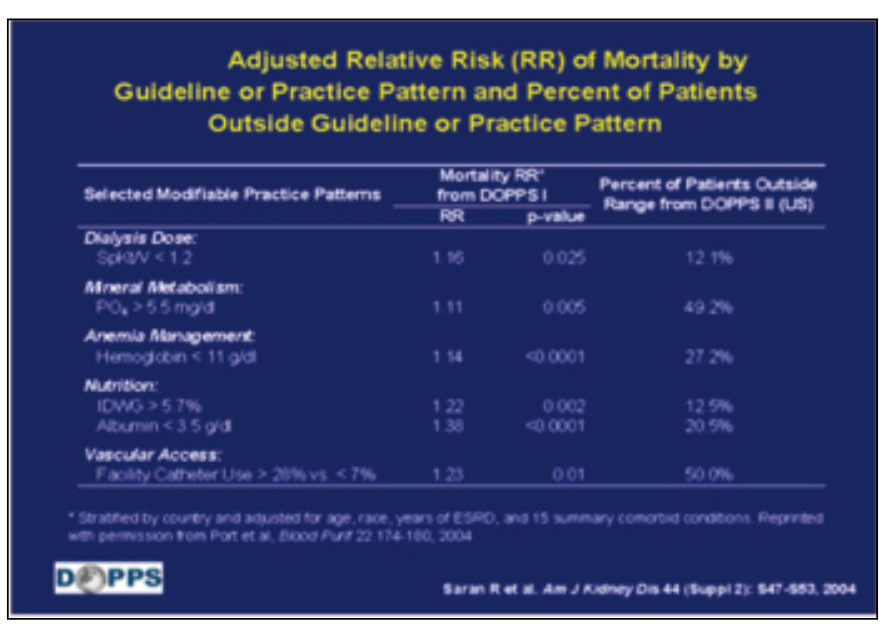

Fig. 3 - Rischio Relativo di morte in relazione alla percentuale di pazienti con valori al di fuori delle linee guida in relazione a dose dialitica, metabolismo minerale, gestione dell'anemia, nutrizione come aumento interdialitico di peso e livelli di albumina ed accesso vascolare.

$\mathrm{Qb}$ bassi nella popolazione giapponese possono essere giustificati dalla taglia medio-piccola della popolazione, dalle abitudini alimentari, dalla cultura della disciplina applicata che si esplica come maggiore aderenza alle prescrizioni (7). A parità di durata di dialisi troviamo infatti $\mathrm{Qb}$ maggiori nella popolazione americana e nordeuropea dove le taglie XL sono la normalità, le proteine animali una consolidata abitudine alimentare e, non da ultimo, il senso della disciplina socialmente meno radicato.

I dati DOPPS confermano che l'aderenza alle linee gui$\mathrm{da}$, in generale e nello specifico del KT/V, dimunisce la mortalità e la morbidità. La sottostima della dose, e/o della sua erogazione, è più critica nei soggetti maschili, giovani e di taglia medio-grande. In questi soggetti le strategie di erogazione della dose dialitica (attraverso il $\mathrm{Qb}$, lo stato dell'accesso vascolare, la durata del trattamento, il filtro ecc) devono essere monitorate ed adeguate ai cambiamenti fisiologici o patologici. Nei soggetti femminili il conseguimento della dose dialitica è in qualche modo più facilmente raggiungibile all'interno dello standard dialitico (3). Le donne infatti hanno metabolismi basali minori, minore patrimonio muscolare e generalmente hanno minori introiti proteici. Questo facilita l'efficacia della dialisi, tanto che all'aumentare del KT/V si può assistere a un progressivo ridursi del rischio relativo di mortalità (RR), andamento che non è osservato nei soggetti di sesso maschile (3).

Per ovvie ed opposte ragioni a quanto sopra esposto i pazienti anziani che nel tempo cambiano e/o diminuiscono fisiologicamente i loro bisogni metabolici e ali-

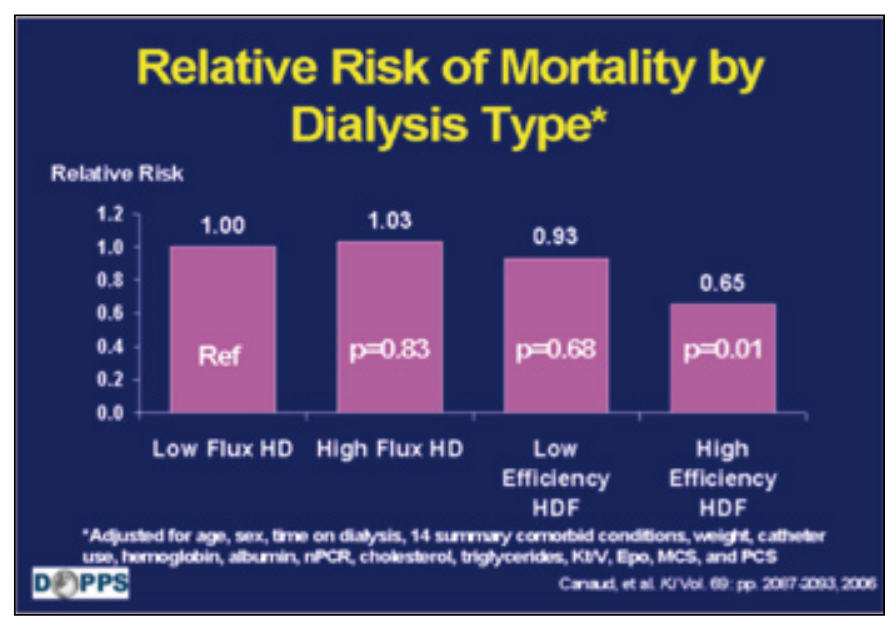

Fig 4 - Rischio relativo di mortalità in base al tipo di trattamento dialitico.

mentari, sono da sorvegliare strettamente dal punto di vista nutrizionale. Buoni valori di $\mathrm{KT} / \mathrm{V}$ infatti possono anche nascondere uno stato di malnutrizione, oppure una FAV che nel tempo perde di efficienza (aneursimi, eventi trombotici ecc). Per queste ragioni il solo KT/V non è esaustivo in senso stretto, ma può dover essere correlato ad altri valori (4).

\section{Altri fattori che concorrono alla efficacia del trattamento}

DOPPS ha correlato il dato del KT/V ad altri 5 parametri di benessere che sono l'accesso vascolare (CVC/ FAV), il metabolismo minerale (Ca, P, PTH), l'anemia $(\mathrm{Hb})$, la nutrizione, includendo albumina e aumento di peso interdialitico (4). Con un adeguato calcolo statistico i dati sono stati elaborati per evidenziare quanto ogni singolo fattore incida sul Rischio Relativo di mortalità (RR) e quanti soggetti del campione analizzato fossero ancora fuori dai parametri indicati dalle linee guida dei KDOQI. Nella Figura 3 sono elencati in colonna i fattori considerati, a seguire il rischio di mortalità, quindi la significatività del dato e la percentuale di pazienti i cui dati erano al di fuori dei range indicati dalle linee guida (LG). Prendendo come valore di RR 1,00 tutte le cifre superiori indicano un aumento del rischio di mortalità. Nella Figura i valori maggiori sono corrispondenti ai fattori nutrizionali e alla presenza di CVC. Si noti come a fronte del fatto che solo il $12,4 \%$ del campione non rientrasse nel range indicato dalle $\mathrm{LG}$ rispetto al raggiungimento 
del KT/V, ben il 49,2\% ne era al di fuori relativamente al metabolismo del fosforo, il $50 \%$ rispetto alla percentuale consigliata di pazienti che usano un CVC come accesso vascolare per dialisi, il $27,2 \%$ rispetto al target per la emoglobina e il 20,5\% rispetto all'apporto proteico. Tutto ciò ovviamente è un chiaro indice di come ancora in alcune aree geografiche vi sia spazio per mitigare il rischio di mortalità e/o morbidità.

Un altro ambito in cui DOPPS ha raccolto dati interessanti è quello dei trattamenti ad alta efficienza, siano essi di sola HD che di HDF. I dati attestano una minore mortalità per i trattamenti ad alta efficienza, in particolare per il trattamento di HDF in cui alla depurazione diffusiva viene associata una personalizzabile quota convettiva. Questi trattamenti sono disponibili solo in Europa, per cui i dati di confronto sono tra i vari Stati europei. Di primo acchito si potrebbe concludere che i dati di minore mortalità nell'HDF siano da correlare alle migliori condizioni generali dei pazienti che sono sottoposti ad HDF. In realtà i dati della Figura 4 sono stati già ricalcolati in base alle 14 variabili di comorbidità normalmente considerate in tutti i dati DOPPS: nonostante ciò il dato di minore mortalità è significativo. Secondo gli esperti, ciò che maggiormente concorre alla minore mortalità, pare essere la componente convettiva del trattamento, che permette la rimozione dei soluti di peso molecolare medio alto (9).

\section{Discussione}

A fronte di una maggiore attenzione clinica al raggiungimento del KT/V, ampiamente verificato, restano critiche alcune importanti aree in cui l'intervento infermieristico ha un impatto significativo: metabolismo minerale, accesso vascolare e nutrizione.

Il metabolismo minerale necessita di informazione, educazione, consapevolezza e sostegno del paziente rispetto all'importanza della dieta, dell'assunzione delle terapie fosfo-chelanti, delle implicazioni fisiopatologiche innescate dal dismetabolismo minerale. La consulenza e il supporto informativo reiterato, in concomitanza degli esami mensili, sono modalità fondamentali perché il paziente riesca a mantenere una buona compliance, adeguando il proprio stile ed abitudini di vita.

Laccesso vascolare, qui visto come presenza di Centri in cui la percentuale di CVC rispetto alla FAV è superiore ai range consigliati, ha anch'esso una notevole correlazione con l'assistenza sia in dialisi sia nel pre-dialisi. In altro articolo è stato evidenziato come il CVC abbia un rischio di mortalità da 5 a 8 volte superiore alla fistola arterovenosa. Nel pre-dialisi è importante programmare il confezionamento precoce della FAV (6), permettendo un tempo adeguato di maturazione, prima dell'inizio del trattamento. In dialisi è responsabilità infermieristica gestire e monitorare il buon funzionamento della FAV attraverso un ottimale esame obiettivo prima di ogni seduta dialitica sia nelle fasi iniziali quando la fragilità vasale è ancora notevole (10), sia nel prosieguo quando le complicanze dell'accesso vascolare nativo o protesico tendono a manifestarsi. Investire energie organizzative e professionali nelle prime fasi, scegliendo ed applicando idonee strategie di puntura e una oculata gestione dei primi trattamenti dialitici permette di evitare danni operativi, dolori al paziente, costi per l'organizzazione. In fase di trattamento cronico è altrettanto importante il buon monitoraggio della FAV e, anzi, stanno emergendo nuovi strumenti di monitoraggio. Tra questi vi è una procedura alquanto semplice ed affidabile per monitorare la portata di una FAV specie se nativa. Il test studiato nel nostro Paese denominato "QB stress test" sembra affidabile specialmente per la ricerca degli eventi stenotici perianastomotici ed è di semplice esecuzione e quindi facilmente ripetibile anche in concomitanza dei consueti controlli ematici mensili (11).

L'osservazione delle condizioni nutrizionali dei pazienti è anch'essa di primaria importanza, soprattutto in presenza di pazienti anziani, in concomitanza con eventi patologici acuti o cronici. Una precoce rilevazione delle principali alterazioni può attivare in tempo consulenze dietologiche, supporti specifici e scelte condivise con pazienti e care givers. Anche la perdita di peso durante la seduta dialitica ha un impatto sui dati di mortalità: minori quote di ultrafiltrazione oraria sono correlate a minore rischio di mortalità (12). Lattenzione assistenziale deve però tenere in grande considerazione il singolo paziente e le sue generali condizioni nutrizionali.

\section{Conclusioni}

Il KT/V è un parametro molto utile, non sempre esaustivo ma di ottimo indirizzo. I DOPPS danno evidenza scientifica a quegli aspetti che spesso ci siamo ritrovati ad affrontare con i nostri pazienti considerandole "chiacchere di buon senso". Oggi possiamo affermare che ci sono evidenze scientifiche per cui lo stile di vita, la tipologia della persona e l'aderenza alle prescrizioni farmacologiche, sono variabili importanti tanto quanto quelle strettamente tecnologiche. Le "chiacchere di buon senso" sono importanti momenti di educazione terapeutica che concorrono a fare la differenza, a trovare la giusta alchimia tra il mandato clinico, i dati tecnici e il benessere percepito dalla persona. La consultazione del sito DOPPS (13), 
completamente accessibile e gratuita, può fornire spunti di approfondimento per professionisti esperti nonché opportunità didattiche per professionisti neofiti.

\section{Indirizzo degli Autori:}

Marisa Pegoraro

Dialisi Cal Corsico

Via Travaglia 5

20094 Corsico (Mi)

marisa_pegoraro@fastwebnet.it

\section{Bibliografia}

1. Lopot F. Urea Kinetic Modelling, EDNTA/ERCA Series, 1990.

2. Port FK, Pisoni RL, Bommer J, Locatelli F, Jadoul M, Eknoyan G, Kurokawa K, Canaud BJ, Finley MP, Young EW. Improving Outcomes for Dialysis Patients in the International Dialysis Outcomes and Practice Patterns Study. Clin J Am Soc Nephrol 2006; 1(2): 246-255.

3. Port FK, Wolfe RA, Hulbert-Shearon TE, Ashby VB, McCullough KP, Held PJ. High dialysis dose is associated with lower mortality among women but not among men. Am J Kidney Dis 2004; 43(6): 1014-23.

4. Saran R, Canaud B, Depner T, Keen M, McCullough KP, Marshall MR, Port FK. Dose of Dialysis: Key Lessons from Major Observational Studies and Clinical Trials. Am J Kidney Dis 2004; 44 (Suppl 2): S47-S53.

5. Mahon A, Jenkins K: CKD stage 3-5, A practical guide for clinical Practice. EDTNA/ERCA , 2008.

6. www.nxstage.com

7. Rayner HC, Pisoni RL, Gillespie BW, et al. Creation, cannulation and survival of arteriovenous fistulae: data from the Dialysis Outcomes and Practice Patterns Study. Kidney Int 2003; 63(1): 323-30.

8. Lopes AA, Bragg-Gresham JL, Elder SJ, et al. Independent and joint associations of nutritional status indicators with mortality risk among chronic hemodialysis patients in the Dialysis Outcomes and Practice Patterns Study (DOPPS). J Renal Nutr 2010; 20(4): 224-34.

9. Canaud B, Bragg-Gresham JL, Marshall MR, Desmeules S, Gillespie BW, Depner T, Klassen P, Port FK. Mortality risk for patients receiving hemodiafiltration versus hemodialysis: European results from the DOPPS. Kidney Int 2006; 69: 2087-93.

10. Goodkin DA, Pisoni RL Locatelli F, Port FK, Saran R. Hemodialysis vascular access training and practices are key to improved access outcomes. Am J Kidney Dis 2010; 56(6): 1032-42.

11. Bonforte G, Pogliani D, Brenna S, et al. Validation of QB stress test a san useful tool in the detection of nativa arteriovenous fistula stenosis: result after 22 months of follow-up. NDT 2010; 11: 1-7.

12. Saran R, Bragg-Gresham JL, Wizemann V, et al. Longer treatment time and slower ultrafiltration in hemodialysis: Associations with reduced mortality in the DOPPS. Kidney Int 2006; 69: 1222-8.

13. www.dopps.org 